\title{
Wie ist die Lage in Einrichtungen für frühkindliche Regulations- störungen in Deutschland?
}

\section{Eine Studie zur Rolle des Schlafes und elterlicher Unsicherheiten sowie angewandter Behandlungskonzepte}

\author{
Marisa Schnatschmidt ${ }^{1}$, Barbara Cattarius ${ }^{1}$, Barbara Schneider ${ }^{2}$, Andreas Jud Ad, $^{3,4}$ \\ und Angelika A. Schlarb ${ }^{1}$
}

${ }^{1}$ Fakultät für Psychologie und Sportwissenschaft, Abteilung für Psychologie, Universität Bielefeld

${ }^{2}$ Zentrum für Neuropädiatrie und Schlafmedizin, Kinderkrankenhaus St. Marien, Landshut

${ }^{3}$ Kinder- und Jugend psychiatrie/Psychotherapie, Universitätsklinikum UIm

${ }^{4}$ Soziale Arbeit, Hochschule Luzern, Luzern, Schweiz

\begin{abstract}
Zusammenfassung: Theoretischer Hintergrund: Frühkindliche Regulationsprobleme stehen in Verbindung mit Schlafproblemen und unsicherem Elternverhalten. Störungsspezifische Behandlungsansätze sind vielfältig und unterscheiden sich deutlich hinsichtlich Qualität und Evidenzgrad. Fragestellung: Häufigkeiten von Schlafproblemen, elterliche Unsicherheiten, therapeutisch / beraterische Grundorientierungen und Konzepte werden erfasst und Unterschiede zwischen Einrichtungsarten untersucht. Methode: Die Umfrage wurde mit interdisziplinären Experten entwickelt. Eine deutschlandweite Datenbank (622 Einrichtungen) mit einer finalen Stichprobe von $N=160$ wurde erstellt. Ergebnisse: Schlafprobleme gelten als häufigster Anmeldegrund (59\%), unsicheres Erziehungsverhalten als mehrheitlich relevant (66\%). Über 16 Behandlungskonzepte werden angewandt; je nach Einrichtungsart eher systemische $(d=0.5)$, körperorientierte $(d=0.5)$ oder somatisch-medizinische $(d=0.7)$ Verfahren. Schlussfolgerung: Die schlafspezifische Expertise der Fachkräfte sollte gesichert sowie Transparenz und Überprüfbarkeit von Qualitätsstandards weiterentwickelt werden.
\end{abstract}

Schlüsselwörter: Frühkindliche Regulationsprobleme, Schlafprobleme, Unsicherheit im Erziehungsverhalten, Behandlungsansätze

The Present Situation in Institutions for Early-Childhood Regulatory Problems: A Study of the Role of Sleep and Uncertainties in Parenting Competence and Suggestions for Treatment

Abstract: Background: Early-childhood regulatory problems are associated with sleep problems and uncertainties in parenting competence. There are various disorder-specific treatment approaches available that differ sharply in terms of quality and level of evidence. Objectives: We assessed the frequency of sleep problems, uncertainties in parenting competence, treatment approaches, and manuals as well as investigating possible differences between types of facilities. Methods: The survey was developed conjointly with interdisciplinary experts. We created a Germany-wide database of 622 institutions with a final sample of $N=160$. Results: Sleep problems are the most common reason for consultation (59\%) and uncertainties in parenting competence as highly relevant $(66 \%)$. We applied more than 16 treatment approaches, depending on the type of facility: systemic $(d=0.5)$, body-oriented $(d=0.5)$, or somatic-medical $(d=0.7)$ procedures. Conclusions: The sleep-specific ability of professionals should be guaranteed as well as the transparency and verifiability of quality standards.

Keywords: early regulatory problems, sleep problems, uncertainties in parenting competence, treatment approaches

In den ersten Lebensjahren manifestieren sich frühkindliche Regulationsstörungen (FRS) hauptsächlich in Schlafstörungen, exzessivem Schreien und Fütterproblemen (Zero to Three, 2016). Dies betrifft etwa $20 \%$ aller Säuglinge im ersten Lebensjahr (Hemmi, Wolke \& Schneider, 2011).
FRS haben langfristige Auswirkungen u. a. auf die Fähigkeit zur Selbstregulation, soziale Fähigkeiten, psychische Gesundheit sowie auf die Eltern-Kind-Beziehung im Kindesalter bis hin zur Adoleszenz (Hemmi et al., 2011; Schmid, Schreier, Meyer \& Wolke, 2010; Winsper \& Wolke, 2014). 


\section{Frühkindlicher Schlaf}

In den ersten Lebensmonaten verändert sich das Schlafverhalten grundlegend. Die Entwicklung eines SchlafWach-Rhythmus stellt daher eine herausfordernde Entwicklungsaufgabe dar, was häufig mit Selbstregulationsproblemen im Säuglings- und Kleinkindalter einhergehen kann (z. B. Schlarb \& Schneider, 2018; Schnatschmidt \& Schlarb, 2018). So werden Schlafprobleme bei Säuglingen als Indikator für Regulationsstörungen betrachtet (Schmid et al., 2010). Schlafprobleme können auch anderen FRS zugrunde liegen und mit ihnen zusammen auftreten. Beispielsweise verhindert ein hohes Erregungsniveau bei Kindern tiefen und kontinuierlichen Nachtschlaf, was zu Tagesschläfrigkeit führt, die wiederum Reizbarkeit und Regulationsprobleme verstärkt (DeGangi, Breinbauer, Roosevelt, Porges \& Greenspan, 2000). Die Bewältigung frühkindlicher Schlafprobleme ist von verschiedenen Faktoren, wie dem Temperament des Kindes, der Fähigkeit zur Selbstberuhigung, aber auch von dem schlafbezogenen Erziehungsverhalten der Eltern abhängig (Cattarius \& Schlarb, 2016; Sadeh et al., 2016).

\section{Erziehungsverhalten}

Die Eltern-Kind-Interaktion ist relevant für die Entwicklung der Fähigkeit zur Selbstregulation und Selbstberuhigung (z.B. Winsper \& Wolke, 2014). Bei FRS kommt es in der Eltern-Kind-Interaktion gehäuft zu einem Teufelskreis, der eine Beruhigung deutlich erschwert (Papoušek \& Wollwerth de Chuquisengo, 2006). Hierin kann das kindliche Schlafverhalten eine Schlüsselrolle einnehmen: häufiges nächtliches Aufwachen des Säuglings führt zu elterlichen Schlafstörungen (z.B. Covington, Armstrong \& Black, 2018), was wiederum mit Tagesauswirkungen seitens der Eltern einhergeht, wie reduzierter Geduld und Gefühlen von Frustration und Reizbarkeit (Nash, Morris \& Goodman, 2008). Zudem führt die Belastung durch FRS bei Kindern zu elterlichen Gefühlen der Hilflosigkeit sowie zu unsicherem und unangemessenem Erziehungsverhalten. Dies kann auch das Risiko für Misshandlung und Vernachlässigung erhöhen, was sich in dieser Altersgruppe insbesondere durch das Shaken Baby Syndrom zeigt (Barr et al., 2009). Daher ist die Forschung zu Behandlungsmöglichkeiten und Anlaufstellen für frühkindliche Schlafprobleme und FRS von hoher Relevanz.

\section{Behandlungsansätze}

Bei FRS kann zwischen den Behandlungsebenen Begleitung, Beratung und Psychotherapie unterschieden wer- den (s. GAIMH Leitlinie, 2005). Eine ähnliche Struktur lässt sich auch bei Weiß und Sann (2013) bezüglich der Organisation der Anlaufstellen der Frühen Hilfen in Deutschland wieder finden. Nach Cierpka, Stasch und Groß (2007) sind die häufigsten therapeutisch / beraterischen Grundorientierung im Eltern-Säuglings-/Kleinkind-Bereich die psychoedukative, die systemische, die kognitiv-verhaltenstherapeutische, die psychodynamische, die körperorientierte, sowie die somatisch-medizinische. In der Praxis ist die Behandlung von FRS von einer Methodenintegration geprägt, so dass Elemente aus den verschiedenen Grundorientierungen häufig in multimodale Behandlungskonzepte integriert werden (z.B. Cierpka, 2014). Daraus ergeben sich häufig Überschneidungen und eine klare Zuordnung zu einer Grundorientierung ist nicht zielführend. Vielmehr können Schwerpunkte der Kernelemente der verschiedenen Konzepte ausgemacht werden.

Konzepte mit einem bindungstheoretischen, interaktionellen Schwerpunkt stellen die Förderung der elterlichen Feinfühligkeit, der positiven Eltern-Kind-Interaktion und einer sicheren Bindungsbeziehung in den Mittelpunkt (siehe z. B. Bolten, 2014). Häufig wird auch mit Videofeedback gearbeitet. $\mathrm{Zu}$ diesem Schwerpunkt zählen beispielsweise folgende Konzepte (Sarimski, 2013): die Integrative ElternSäuglings-Kleinkind-Beratung/-Psychotherapie nach Papoušek (IES / K-B / P; siehe z. B. Papoušek \& Wollwerth de Chuquisengo, 2006), die manualisierte Säuglings- / Kleinkind-Eltern-Psychotherapie (SKEPT; Cierpka, Hirschmüller et al., 2007), die Entwicklungspsychologische Beratung nach Ziegenhain (EPB; Ziegenhain, Fries, Bütow \& Derksen, 2004), das STEEP-Programm (Steps Toward Effective and Enjoyable Parenting; Egeland \& Erickson 2004), die Marte-Meo-Methode (Bünder \& Sirringhaus-Bünder 2008), das SAFE-Programm (Sichere Ausbildung für Eltern; Brisch, 2010) und das ABC-Programm (Attachment and Biobehavioral Catch-up; Dozier \& Bernard, 2017).

Darüber hinaus lassen sich Konzepte mit einem körperorientierten Schwerpunkt ausmachen. In diesen Ansätzen gilt der Körper als das wichtigste Referenzsystem und es wird beispielsweise mit Berührungen und entspannenden Massagetechniken gearbeitet. Weitere Elemente sind unter anderem die bioenergetischen Lehren Wilhelm Reichs oder auch das Aufgreifen prä- und perinataler Dimensionen, Harms, T. (2016a). Dieser Schwerpunkt lässt sich beispielsweise in folgenden Konzepten finden (Harms, 2016a): die Emotionelle Erste Hilfe, (EEH; Harms, 2016b), die Ressourcen- und Körperorientierte Krisenbegleitung (KKB; Diederichs, 2000), Sanfte Bioenergetik nach Reich, Integration der prä- und perinatalen Erfahrungen nach Castellino, Craniosakral-Therapie (Beschreibungen der Konzepte siehe Harms, 2016a) oder die Physiotherapiekonzepte nach Bobath oder Vojta (siehe z.B. Hammerschmidt \& Koch, 2018). 
Cierpka, Stasch und Kollegen (2007) zeigten, dass bei den Behandler_innen von FRS unterschiedliche Qualifikationen und Grundorientierungen vorhanden sind und nach spezifischen häufig multimodalen Konzepten gearbeitet wird. Ihre Studie ergab, dass die Mehrheit der Behandler_innen (39\%) nach der IES / K-B / P nach Papoušek (Papoušek \& Wollwerth de Chuquisengo, 2006) arbeitete. Ebenfalls sehr verbreitet waren paar- und familientherapeutische Weiterbildungen $(16,5 \%)$ sowie körperpsychotherapeutische Behandlungen (14\%). Nach der EBP (Ziegenhain, Fries et al., 2004) arbeiteten 5,34\% der Behandler_innen. Eine Systemanalyse der interdisziplinären Frühförderung in Bayern (FranzL, 2010) stellte ebenfalls eine Vielzahl von angewandten Behandlungskonzepten fest.

Umsetzung und Qualität der Programme unterscheiden sich jedoch sehr stark und entsprechen weitestgehend bisher einem niedrigem Evidenzgrad, da direkt anwendbare klinische Studien von guter Qualität noch ausstehen oder sich die Empfehlungen auf konsentierten Meinungen aus Expertenkreisen und/oder klinischer Erfahrung anerkannter Autoritäten stützen (vgl. AHRQ Evidenzgrad C; AWMF, 2015; Taubner, Munder, Unger \& Wolter, 2013). Eine Herausforderung in diesem Zusammenhang ist die Verbindung der verschiedenen Berufsgruppen, die am multiprofessionellen Ansatz der Frühintervention beteiligt sind und oft in interdisziplinären Teams arbeiten (Ludwig-Körner, 2013). Die letzte störungsspezifische Studie zur Versorgungssituation in Deutschland von Cierpka, Stasch und Kollegen (2007) liegt bereits vierzehn Jahre zurück.

\section{Ziel der Studie}

Aus oben genannten Gründen liegt nahe, in Anlehnung an Cierpka, Stasch und Kollegen (2007), Aspekte der Versorgungsqualität, vor allem die Behandlungsansätze in Einrichtungen für FRS, zu aktualisieren, im Bezug zu den Faktoren kindlicher Schlaf sowie Unsicherheiten im Erziehungsverhalten zu beleuchten und Unterschiede zwischen Einrichtungsarten zu untersuchen. Für die vorliegende Studie wurden daher folgende Forschungsfragen formuliert: Wie häufig sind in Einrichtungen für FRS 1) kindliche Schlafprobleme Anmeldegrund? 2) werden Unsicherheiten im Erziehungsverhalten genannt oder festgestellt? 3) nach welchen therapeutischen/beraterischen Grundorientierungen wird gearbeitet? 4) Welche Behandlungskonzepte/-manuale werden angewandt? 5) Bestehen Unterschiede hinsichtlich Grundorientierung und Behandlungskonzepten zwischen den Einrichtungsarten?

\section{Material und Methoden}

\section{Durchführung}

In Anlehnung an Cierpka, Stasch und Kollegen (2007) wurde für diese Studie eine deutschlandweite Online-Datenbank mit Anlaufstellen für FRS erstellt. Die verwendeten Suchbegriffe waren „Schreiambulanz“, „Babyambulanz“, „Babysprechstunde“, „Schreibaby“, „Regulationsprobleme“, „Regulationsstörungen“. Das Suchinstrument „Ambulante Schreiambulanzen" des Nationalen Zentrums für frühe Hilfen wurde zur Vervollständigung der aktuellen Datenbasis für die vorliegende Studie zusätzlich mit einbezogen. Die finale Datenbank umfasste 622 Einrichtungen (davon 459 Beratungsstellen, 68 Praxen und 95 klinische Einrichtungen). Einschlusskriterium war eine funktionierende E-Mail-Adresse. Daher mussten 14 Einrichtungen ausgeschlossen werden. Die E-Mail zur Kontaktaufnahme mit den Einrichtungen enthielt neben dem Zugang zur Umfrage detaillierte Informationen zu Forschungszielen, Freiwilligkeit der Teilnahme, Datenhandling und Anonymisierung der Umfragedaten. Zusätzlich wurde das Ausfüllen im Papier-Format ermöglicht und nach zwei Wochen wurde eine Erinnerungs-E-Mail verschickt. Die Studie wurde von der Ethikkommission der Universität Bielefeld genehmigt.

\section{Teilnehmende}

Von der ursprünglichen Datenbank mit 622 Einrichtungen erhielten wir 218 (online: 187, Papier: 31) Fragebögen zurück, was einer Rücklaufquote von $35 \%$ entspricht. Davon waren 58 Online-Fragebögen jedoch aufgrund fehlender Angaben nicht auswertbar, sodass die finale Stichprobe $N=160$ Datensätze umfasste. In den einbezogenen Einrichtungen gehörten die meisten Mitarbeitenden den folgenden Berufsgruppen an: Sozialpädagog_innen, Psycholog_innen, Mediziner_innen und Pädagog_innen (s. Abbildung 1). Insgesamt arbeiteten 126 Einrichtungen $(79 \%)$ mit interdisziplinären Teams. Für eine detailliertere Analyse wurden die Einrichtungen in Anlehnung an Weiß und Sann (2013) nach der Struktur der Institutionen der frühen Hilfen in Deutschland kategorisiert. So wurde unterschieden in regionale Anlaufstellen (wie z. B. Beratungsstellen, Familienberatungsstellen, Familienbildungszentren, Kinderschutzzentren verschiedener Anbieter wie Frühförderung, pro familia, Caritas, Diakonie oder kommunale Anbieter), niedergelassene Praxen und an Kliniken angebundene Einrichtungen (wie z.B. Sozialpädiatrische Zentren, spezialisierte Kliniken, Fachabteilungen). Daraus ergab sich für die vorliegende 


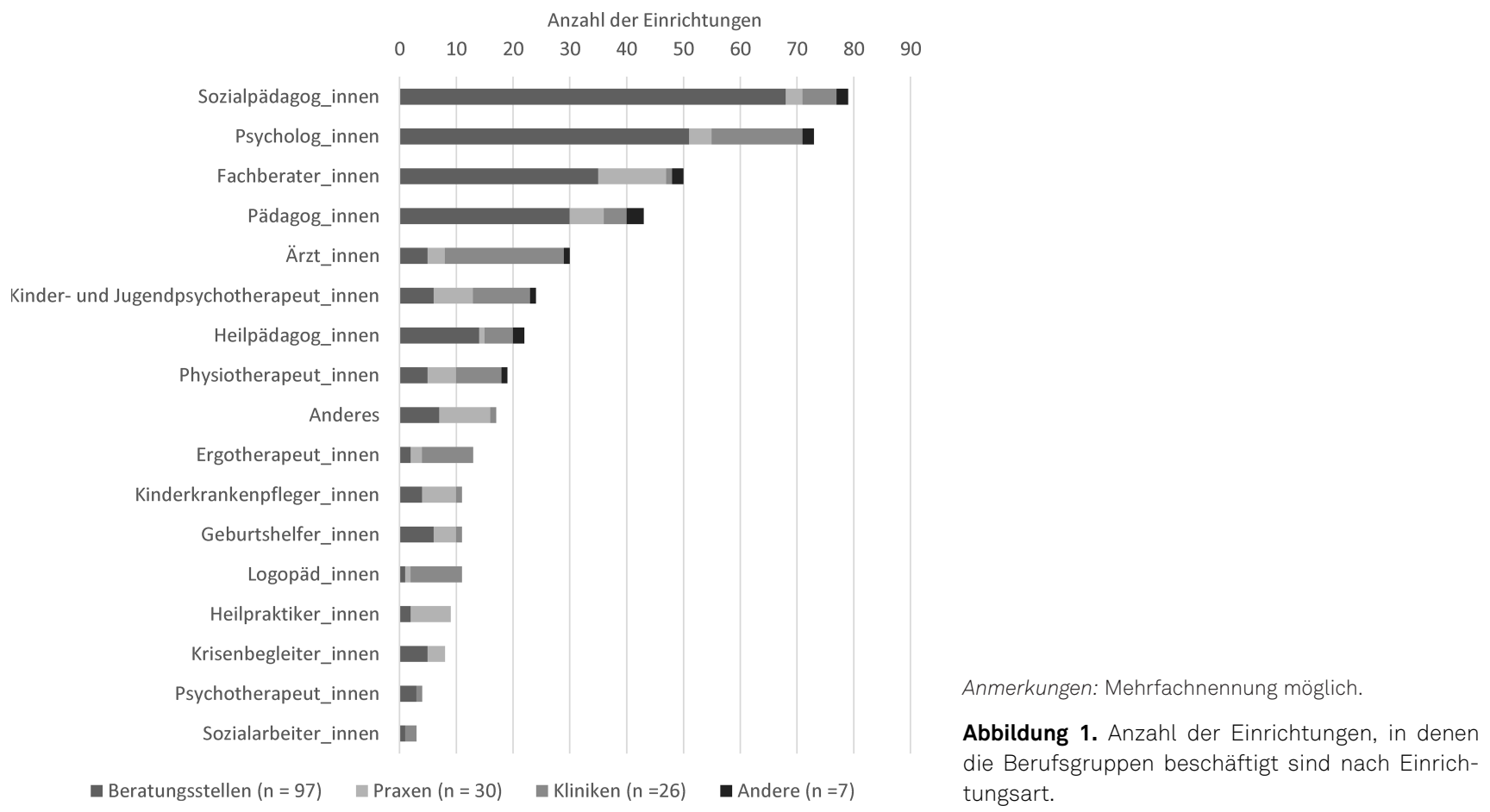

Studie folgende Kategorisierung: Beratungsstellen $(n=97)$, Praxen $(n=30)$, Kliniken $(n=26)$ und andere $(n=7)$.

\section{Instrumente}

Auf der Grundlage früherer Studien zur Versorgungssituation und Prozessqualität im Bereich der Eltern-KindBeratung und Therapie sowie Früher Hilfen in Deutschland (Cierpka, Stasch et al., 2007; FranzL, 2010) sowie multiplen Expertenratings, wurden in vorliegender Studie diverse Bereiche erfasst: 1) Merkmale der Einrichtung (z. B. Art der Einrichtung, Bundesland, Merkmale der Klientel, Art der Abrechnung, Kooperationsstrukturen, Diagnose- und Therapiemodalitäten; 13 Items), 2) Merkmale des Personals (z. B. Berufsgruppe, Art und Zusammensetzung der Teams; 3 Items) und 3) Merkmale der Beratung / Therapie (z. B. Kontext des Anmeldung/der Überweisung, Anmeldegrund, Arbeitsmethoden, Behandlungskonzept, Anzahl der Kontakte, Jahresdurchlauf; 8 Items). Zur Erfassung der angewandten Behandlungsansätzen bei FRS konnten die Teilnehmenden aus den am häufigsten vorkommenden Verfahren (Cierpka, Stasch et al., 2007; FranzL, 2010) ihren angewandten Behandlungsansatz auswählen oder die von ihnen eingesetzten Behandlungsansätze benennen. Mehrfachantworten waren möglich. Insgesamt umfasste der Fragebogen 24 Items und wurde als Online-Befragung in Unipark erstellt (Questback $\mathrm{GmbH}$, 2017).

\section{Analyse}

Zur statistischen Analyse wurden nichtparametrische Kruskal-Wallis-Tests und Wilcoxon-Tests berechnet, da eine Normalverteilung der Daten nicht angenommen werden konnte. Paarweise Vergleiche wurden mittels MannWhitney U-Test berechnet und nach Bonferroni korrigiert. Zur Einordnung der Ergebnisse wurde die Effektstärke $d$ von Cohen (Cohen, 1988) berechnet (kleiner Effekt: $d \geq 0.20$, mittlerer Effekt: $d \geq 0.50$, großer Effekt: $d \geq .80$ ). Die Analysen wurden mit dem sozialwissenschaftlichen Statistikpaket SPSS 27.0 für Windows (IBM, 2020) berechnet.

\section{Ergebnisse}

\section{Schlaf im Kontrast zu anderen FRS}

Die angegebenen Häufigkeiten von FRS (wie Schlafprobleme, übermäßiges Schreien oder Fütterprobleme) als Gründe für die Anmeldung werden in Tabelle 1 dargestellt. Es zeigt sich, dass Schlafprobleme in Beratungsstellen, Praxen und Kliniken den am häufigsten angegebenen Anmeldegrund darstellen.

Für die Häufigkeit von Schlafproblemen und übermäßigem Weinen wurden keine signifikanten Unterschiede zwischen den Einrichtungen festgestellt. Hingegen 
Tabelle 1. Mittelwert und Standardabweichung der Anmeldegründe in Prozent

\begin{tabular}{lcccc}
\hline & $\begin{array}{c}\text { Beratungsstellen } \\
(n=96)\end{array}$ & $\begin{array}{c}\text { Praxen } \\
(n=29)\end{array}$ & $\begin{array}{c}\text { Kliniken } \\
(n=25)\end{array}$ & $\begin{array}{c}\text { Andere } \\
(n=7)\end{array}$ \\
\hline SP & $59.88(24.81)$ & $65.17(22.83)$ & $57.68(23.67)$ & $35.71(28.05)$ \\
ES & $37.98(26.48)$ & $46.14(26.11)$ & $38.88(21.42)$ & $50.00(33.30)$ \\
FP & $13.21(14.22)$ & $20.48(18.26)$ & $23.52(16.61)$ & $6.43(4.76)$ \\
A & $17.16(20.06)$ & $15.45(20.78)$ & $12.48(18.83)$ & $5.00(11.18)$ \\
\hline
\end{tabular}

Anmerkungen: M (SD in Klammern). Die Prozentwerte addieren sich zum Teil nicht zu $100 \%$ aufgrund von Mehrfachnennungen. SP: Schlafprobleme, ES: Exzessives Schreien, FP: Fütterprobleme, A: Anderes (z. B. Trotzen, Unruhe, Ärger/Stress/Aggression, Spielunlust, Trennungsschwierigkeiten/-angst)

Tabelle 2. Ergebnisse des Wilcoxon Tests für Schlafproblemen im Kontrast zu anderen FRS als Anmeldegrund

\begin{tabular}{|c|c|c|c|c|c|c|c|c|}
\hline & \multicolumn{2}{|c|}{$\begin{array}{c}\text { Beratungsstellen } \\
\quad(n=96)\end{array}$} & \multicolumn{2}{|c|}{$\begin{array}{l}\text { Praxen } \\
(n=29)\end{array}$} & \multicolumn{2}{|c|}{$\begin{array}{l}\text { Klinken } \\
(n=25)\end{array}$} & \multicolumn{2}{|c|}{$\begin{array}{l}\text { Andere } \\
(n=7)\end{array}$} \\
\hline & $z$ & $d$ & $z$ & $d$ & $z$ & $d$ & $z$ & $d$ \\
\hline$S P-E S$ & $-5.87 * \star$ & 1.50 & $-2.99 *$ & 1.33 & $-3.10 *$ & 1.58 & -.95 & 0.77 \\
\hline$S P$ - FP & -8.11 ** & 2.95 & $-4.56^{\star \star}$ & 3.18 & $-3.84 * \star$ & 2.40 & -2.21 & 3.03 \\
\hline$S P-A$ & $-7.96 * *$ & 2.78 & $-4.49 * *$ & 3.02 & $-3.60 * *$ & 2.01 & -2.23 & 3.13 \\
\hline
\end{tabular}

Anmerkungen: ${ }^{\star} p<.05,{ }^{\star *} p<.001$. FRS: Frühkindliche Regulationsstörungen, SP: Schlafprobleme, ES: Exzessives Schreien, FP: Fütterprobleme, A: Anderes

Tabelle 3. Mittelwerte und Standardabweichungen der Unsicherheiten im Erziehungsverhalten in Prozent

\begin{tabular}{lcccc}
\hline & $\begin{array}{c}\text { Beratungsstellen } \\
(n=97)\end{array}$ & $\begin{array}{c}\text { Praxen } \\
(n=30)\end{array}$ & $\begin{array}{c}\text { Kliniken } \\
(n=26)\end{array}$ & $\begin{array}{c}\text { Andere } \\
(n=7)\end{array}$ \\
\hline Äußerungen der Eltern & $65.12(27.52)$ & $67.00(24.87)$ & $70.19(25.67)$ & $40.29(36.80)$ \\
Beobachtungen des Fachpersonals & $65.73(24.50)$ & $64.13(23.57)$ & $74.46(21.46)$ & $59.14(38.19)$ \\
\hline
\end{tabular}

Anmerkungen: M (SD in Klammern). Angaben sind Schätzungen des Fachpersonals.

zeigte sich für Fütterprobleme ein signifikanter Unterschied $\left(x^{2}(3, N=144)=20.51, p \leq .001, d=0.77\right)$. In Kliniken waren diese signifikant häufiger der Anmeldegrund als in Beratungsstellen $(z=3.69, p \leq .001, d=0.71)$. Die Ergebnisse bezüglich der Häufigkeiten von Schlafproblemen als Anmeldegrund im Gegensatz zu anderen Regulationsstörungen finden sich in Tabelle 2. Insgesamt wurden Schlafprobleme in Beratungsstellen, Praxen und Kliniken signifikant häufiger als Anmeldegrund angegeben als exzessives Schreien, Fütterprobleme oder andere FRS.

\section{Unsicherheiten im Erziehungsverhalten}

Zur Analyse der Unsicherheiten im Erziehungsverhalten finden sich in Tabelle 3 die Einschätzungen des Fachpersonals bezüglich der beobachteten und von den Eltern kommunizierten Unsicherheiten. Es war kein signifikanter Unterschied in den beobachteten $\left(x^{2}(3, N=157)=4.07\right.$, $p=.253, d=0.32)$ und den kommunizierten $\left(x^{2}(3, N=157)\right.$ $=3.64, p=.302, d=0.30)$ Unsicherheiten zwischen den verschiedenen Arten der Einrichtungen festzustellen. Auch in der Gesamtstichprobe unterschieden sich die geschätzten Häufigkeiten der Äußerungen der Eltern $(M=65.21$, $S D=27.51)$ und der Beobachtungen des Fachpersonals $(M=66.56, S D=24.60)$ hinsichtlich der Unsicherheiten im Erziehungsverhalten nicht signifikant voneinander (asymptotischer Wilcoxon Test für eine Stichprobe: $z=-0.78, p=.425, d=0.12, N=160$ ).

\section{Therapeutische / beraterische Grundorientierungen}

In Anlehnung an Cierpka, Stasch und Kollegen (2007) wurden die Angaben den häufigsten therapeutische / beraterischen Grundorientierung im Eltern-Säuglings- / Kleinkind-Bereich zugeordnet: der psychoedukativen, der systemischen, der kognitiv-verhaltenstherapeutischen, der psychodynamischen, der körperorientierten, der humanistischen sowie der somatisch-medizinischen Grundorientierung. Die Mehrheit der Einrichtungen für FRS arbeiteten 


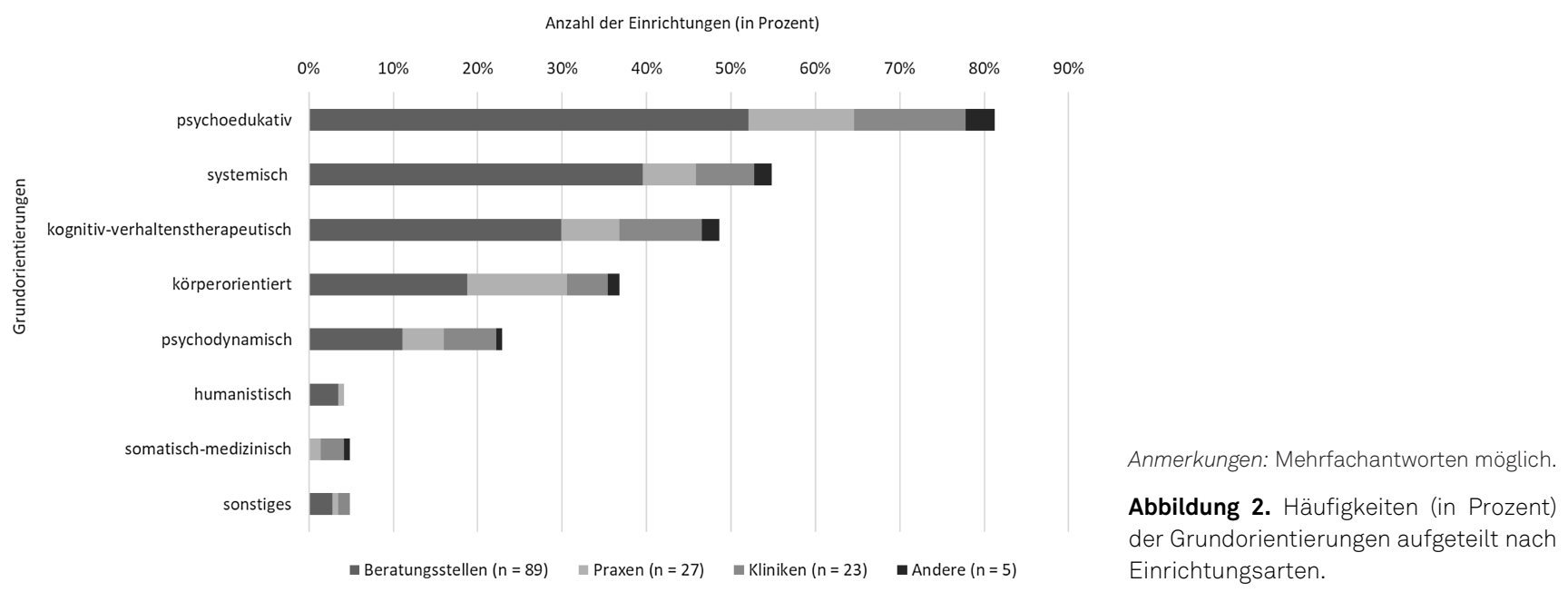

nach mehr als einer Grundorientierung ( $n=106,66 \%)$, 24 Einrichtungen (15\%) arbeiteten nach einer Grundorientierung und 14 Einrichtungen (9\%) boten keine Behandlung an. Diesbezüglich konnten keine signifikanten Unterschiede zwischen den Einrichtungsarten festgestellt werden. Bei 16 Einrichtungen fehlten die Angaben (10\%). Die am häufigsten genannten Grundorientierungen waren die psychoedukative, gefolgt von der systemischen und der kognitiv-verhaltenstherapeutischen sowie der körperorientierten (siehe Abbildung 2). Signifikante Unterschiede zwischen den Einrichtungsarten konnten für die systemische $\left(x^{2}(3, N=144)=9.28, p=.026, d=0.52\right)$, die körperorientierte $\left(x^{2}(3, N=144)=9.99, p=.019, d=0.55\right)$ und die somatisch-medizinische $\left(x^{2}(3, N=144)=14.77\right.$, $p=.002, d=0.68)$ Grundorientierung festgestellt werden. So wurde in Beratungsstellen signifikant häufiger nach der systemischen Grundorientierung gearbeitet als in Praxen $(z=2.80, p=.031, d=0.48)$. Nach der körperorientierten wurde signifikant häufiger in Praxen als in Beratungsstellen $(z=3.07, p=.013, d=0.53)$ und nach der somatisch-medizinischen Grundorientierung an klinischen Einrichtungen signifikant häufiger als in Beratungsstellen $(z=3.71, p \leq .001, d=0.65)$ gearbeitet.

\section{Behandlung der FRS nach Konzepten oder Manualen}

Bezüglich der Anzahl der angewandten Behandlungskonzepte gaben 80 Einrichtungen $(50 \%$ ) an, nach einem und 22 Einrichtungen (14\%) nach mehr als einem Behandlungskonzept $\mathrm{zu}$ arbeiten, während 42 Einrichtungen $(26 \%)$ kein Behandlungskonzept verfolgten. Zwischen den Arten der Einrichtungen konnten diesbezüglich keine signifikanten Unterschiede festgestellt werden. Die vier häufigsten angewandten Behandlungskonzepte werden in Tabelle 4 kurz beschrieben. Die Anwendung dieser Behandlungskonzepte ist in Abbildung 3 dargestellt. Darüber hinaus wurden insgesamt fünf Konzepte mit einem eher bindungstheoretischen, interaktionellen Schwerpunkt angewandt (SKEPT: $n=5$; STEEP: $n=1$; Marte-Meo: $n=3$; SAFE: $n=3$; ABC: $n=1$ ), während zusätzlich zwei schlafspezifische verhaltenstherapeutisch orientierte Konzepte (Mini-KiSS: $n=2$; Freiburger Training: $n=1)$ und nicht näher beschriebene psychodynamische Konzepte $(n=2)$ genannt wurden. Weitere vier Konzepte konnten einem eher körperorientierten Schwerpunkt (Integration der prä- und perinatalen Erfahrungen, Castellino: $n=2$; sanfte Bioenergetik, Reich: $n=1$; Craniosakral-Therapie: $n=1$; Physiotherapiekonzepte, Bobath: $n=1)$ zugeordnet werden. Zudem wurden sechs eigene Ausarbeitungen genannt und in acht Fällen wurden die genannten Konzepte nicht näher spezifiziert, so dass keine klare Zuordnung erfolgen konnte. Insgesamt konnten also neben den sechs eigenen Ausarbeitungen 16 Konzepte identifiziert werden, die zur Behandlung der FRS angewandt wurden.

\section{Diskussion}

In Einrichtungen für FRS sind kindliche Schlafprobleme im Gegensatz zu anderen FRS der häufigste Anmeldegrund. Zudem berichtet das Fachpersonal bei mehr als der Hälfte der vorstelligen Familien von Unsicherheiten im Erziehungsverhalten. Oft sind in einer Einrichtung mehrere therapeutische/beraterische Grundorientierungen vertreten, was die hohe Interdisziplinarität des Fachpersonals widerspiegelt und sich auch nach Einrichtungsart unterscheidet. Die Mehrheit der Einrichtungen arbeitet nach bestimmten Konzepten oder Manualen zur Be- 
Tabelle 4. Wesentliche Merkmale der am häufigsten angewandten Behandlungskonzepte

\begin{tabular}{|c|c|c|c|}
\hline Behandlungskonzepte & Ziele & Grundelemente & Umfang \\
\hline $\begin{array}{l}\text { Integrative Eltern-Kind-Be- } \\
\text { ratung / Psychotherapie nach } \\
\text { Papoušek }{ }^{1,2} \text { (IES/K-B/P) }\end{array}$ & $\begin{array}{l}\text { möglichst rasche Behebung der Re- } \\
\text { gulationsstörung, wirksame Entlas- } \\
\text { tung der Eltern, Unterstützung posi- } \\
\text { tiver Interaktionen und Beziehungs- } \\
\text { erfahrungen }\end{array}$ & $\begin{array}{l}\text { Entwicklungsberatung, psychothera- } \\
\text { peutische Gespräche, (videogestütz- } \\
\text { te) Kommunikationsanleitung, bei } \\
\text { Bedarf Ergänzung psychodynami- } \\
\text { scher Kommunikations- und Bezie- } \\
\text { hungstherapie }\end{array}$ & $\begin{array}{l}\text { individuell auf die Symptomatik, die } \\
\text { psychosoziale Situation und die Be- } \\
\text { dürfnisse der jeweiligen Familie ab- } \\
\text { gestimmt }\end{array}$ \\
\hline $\begin{array}{l}\text { Entwicklungspsychologische } \\
\text { Beratung nach Ziegenhain } \\
\text { (EPB) }\end{array}$ & $\begin{array}{l}\text { Förderung elterlicher Feinfühligkeit, } \\
\text { Förderung einer sicheren Bindungs- } \\
\text { beziehung, Stärkung des Selbst- } \\
\text { wertgefühls der Bezugsperson, Be- } \\
\text { tonung der individuellen Fähigkeiten } \\
\text { des Kindes, langfristige Prävention } \\
\text { von Entwicklungsstörungen und Ver- } \\
\text { haltensauffälligkeiten }\end{array}$ & $\begin{array}{l}\text { lösungsorientiertes Anamnesege- } \\
\text { spräch, Vermittlung von entwick- } \\
\text { lungspsychologischem Wissen, res- } \\
\text { sourcenorientierte Videoanalysen } \\
\text { (Sehen - Verstehen - Handeln), Ge- } \\
\text { spräche über Befindlichkeiten der } \\
\text { Eltern und Alltagsstrukturierung }\end{array}$ & $\begin{array}{l}\text { in der Regel } 5-7 \text { Termine, bei Bedarf } \\
\text { können weitere vereinbart werden }\end{array}$ \\
\hline $\begin{array}{l}\text { Ressourcen und Körperori- } \\
\text { entierte Krisenbegleitung } \\
\text { nach Diederichs }\end{array}$ & $\begin{array}{l}\text { Schaffung von Ruhe und Akzeptanz, } \\
\text { ressourcenorientierte Stärkung der } \\
\text { Elternkompetenz, Reduktion des } \\
\text { Stressniveaus der Krisensituation }\end{array}$ & $\begin{array}{l}\text { Arbeit am Organismus des Kindes } \\
\text { (Massagen, Halte- und Beruhigungs- } \\
\text { übungen), beraterisch-therapeuti- } \\
\text { sche Gespräch mit der Bezugsperson } \\
\text { (klärungs- und bewältigungsorien- } \\
\text { tiert), Arbeit am Organismus der Be- } \\
\text { zugsperson (Halte-, Atemübungen, } \\
\text { Massagen) }\end{array}$ & $\begin{array}{l}\text { variiert je nach Problematik, in der } \\
\text { Regel 5-10 Sitzungen }\end{array}$ \\
\hline $\begin{array}{l}\text { Emotionelle Erste Hilfe nach } \\
\operatorname{Harms}^{6}(\mathrm{EEH})\end{array}$ & $\begin{array}{l}\text { Verbesserung des Bindungserlebens } \\
\text { zum Kind, Verbesserung der allge- } \\
\text { meinen Orientierung im Lesen der } \\
\text { kindlichen Verhaltenssprache, Aus- } \\
\text { bau der elterlichen Fähigkeit zur } \\
\text { Emotionsregulation, Entwicklung } \\
\text { von konkreten Handlungsmöglich- } \\
\text { keiten im Umgang mit den kindlichen } \\
\text { Verhaltensauffälligkeiten }\end{array}$ & $\begin{array}{l}\text { Atmung, Selbstanbindung, Körper- } \\
\text { berührungen, Visualisierungen, Pro- } \\
\text { zess nach dem 7-Schritt-Modell }\end{array}$ & $\begin{array}{l}\text { variiert je nach Anwendungsfeld: } \\
\text { Bindungsförderung, Kriseninterven- } \\
\text { tion (in der Regel } 3 \text { bis } 6 \text { Sitzungen) } \\
\text { und Eltern-Säugling-Bindungspsy- } \\
\text { chotherapie }\end{array}$ \\
\hline
\end{tabular}

Anmerkungen: Eigene Zusammenstellung anhand folgender Quellen. 1) Wollwerth de Chuquisengo \& Papoušek, 2004; 2) Cierpka, 2014; 3) Ziegenhain, Fries, Bütow, \& Derksen, 2004; 4) Diederichs, 2000; 5) Diederichs, 2021; 6) Harms, $2016 \mathrm{~b}$.

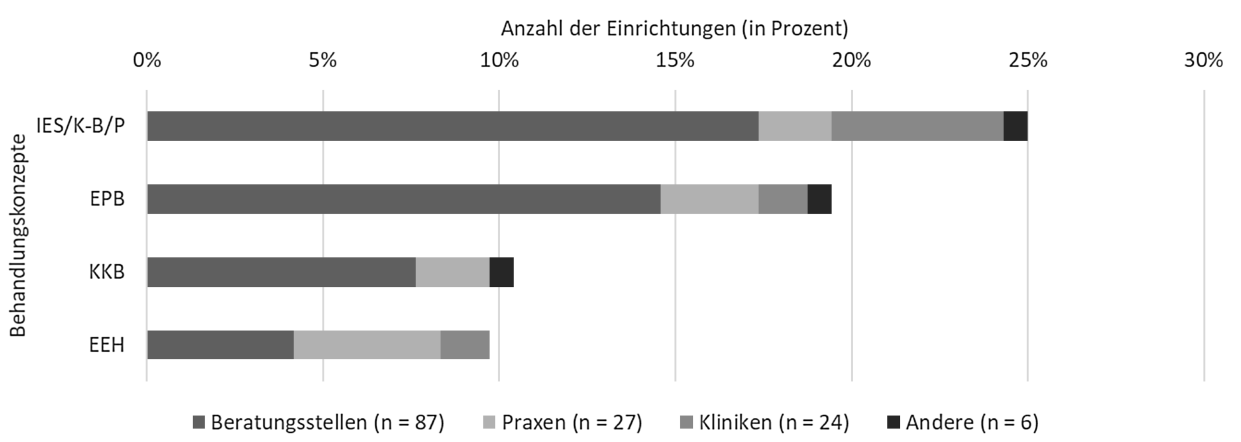

Anmerkungen: Antworten im offenen Antwortformat, Mehrfachnennungen möglich. IES/K-B/P: Integrative Eltern-Kind-Beratung/Psychotherapie nach Papoušek; EPB: Entwicklungspsychologische Beratung nach Ziegenhain; KKB: Ressourcen und Körperorientierte Krisenbegleitung nach Diederichs; EEH: Emotionelle Erste Hilfe nach Harms.

Abbildung 3. Häufigkeiten (in Prozent) der vier häufigsten angewandten Behandlungskonzepte aufgeteilt nach Einrichtungsargen.

handlung der FRS. Insgesamt wird ein sehr breites Spektrum an Behandlungsansätzen angewandt von denen einige wenige (z. B. Craniosakral-Therapie) auch als jenseits der anerkannten wissenschaftlichen Methoden liegend zu werten sind.

\section{Schlaf im Kontext von FRS}

Schlafprobleme liegen sowohl in Praxen als auch in Kliniken und Beratungsstellen für FRS der Mehrzahl der Anmeldungen zugrunde. Im Vergleich werden sie signifikant häufiger als alle anderen Regulationsstörungen be- 
richtet. Diese Ergebnisse stehen im Einklang mit früherer Forschung von Cierpka, Stasch und Kollegen (2007) sowie der postulierten Prävalenz der FRS (Hemmi et al., 2011). In weiteren Untersuchungen wird auf das häufige gemeinsame Auftreten von Schlaf- und Schreiproblemen insbesondere in den Abend- und Nachtstunden hingewiesen (z. B. Cook, Mensah, Bayer \& Hiscock, 2019). Dies wiederum entspricht den Ergebnissen der aktuellen Studie, dass übermäßiges Schreien schätzungsweise der zweithäufigste Anmeldegrund in den Einrichtungen für FRS ist. Die vorliegenden Ergebnisse deuten darauf hin, dass alle Einrichtungen ein ähnliches Klientel mit ähnlichen Anfragen haben. Nur bei Fütterproblemen scheint eher ein klinisches Setting aufgesucht zu werden. Dies stimmt mit früheren Untersuchungen überein (Bolten, 2013).

\section{Unsicheres Erziehungsverhalten bei FRS}

Sowohl in Beratungsstellen, Praxen als auch Kliniken werden nach den Erfahrungen des Fachpersonals in den meisten Familien Unsicherheiten im Erziehungsverhalten deutlich. Ebenso zeigten frühere Studien einen Zusammenhang zwischen FRS und Unsicherheiten im Erziehungsverhalten (Bilgin \& Wolke, 2017; Cook et al., 2019). Naheliegend ist, dass Kinder mit Regulationsproblemen eine erhöhte Belastung für die Eltern darstellen und die Eltern sich häufig überfordert, frustriert und hilflos fühlen. Zusätzlich können diese Gefühle auf Seiten der Eltern das Erlernen der Regulation für das Kind erschweren (z. B. Nash, Morris \& Goodman, 2008; Papoušek \& Wollwerth de Chuquisengo, 2006). Darüber hinaus konnten wir aufzeigen, dass in der Erfahrung des Fachpersonals diese Unsicherheiten auch von Seiten der Eltern kommuniziert werden. Dies ist laut Spranz-Fogasy (2010) notwendig um einen adäquaten Ansatzpunkt in der Behandlung gewährleisten zu können. Allerdings sind diese Ergebnisse mit Vorsicht zu interpretieren, da beide Werte auf der Einschätzung der Fachkräfte beruhen.

Insgesamt legen die hier dargestellten Ergebnisse nahe, dass Unsicherheiten im Erziehungsverhalten ein möglicher Ansatzpunkt einer Behandlung FRS sind. Dies wird bereits in einigen Behandlungskonzepten berücksichtigt, z.B. in Form von bindungsorientierten Arbeiten (siehe Ludwig-Körner, 2013).

\section{Therapeutische / beraterische Grundorientierungen}

Die Ergebnisse der Studie zeigen, dass die psychoedukative die am häufigsten angewandte Grundorientierung in allen Arten der Einrichtungen ist. Dies scheint plausibel, da Beratungen bei FRS oft schon eine gute Unterstützung für die Familien bieten können (Bolten, 2013). Auch die Ergebnisse von Cierpka, Stasch und Kollegen (2007) zeigen die Vermittlung von eher allgemeinen entwicklungsbezogenen Informationen als wesentliches Element in der Behandlung unabhängig von den potenziell unterschiedlichen Grundorientierungen. Die vorliegenden Ergebnisse zeigen, dass die systemische Grundorientierung überwiegt, gefolgt von der kognitiv-verhaltenstherapeutischen, der körperorientierten und der psychodynamischen Grundorientierung, was mit vorherigen Studien übereinstimmt (Cierpka, Stasch et al., 2007; FranzL, 2010). Hinsichtlich möglicher Unterschiede zwischen den Einrichtungsarten, konnte festgestellt werden, dass nach der systemischen Grundorientierung vor allem in Beratungsstellen und nach der körperorientierten überwiegend in Praxen gearbeitet wird. Nach der somatisch-medizinischen Grundorientierung wird hauptsächlich im klinischen Kontext gearbeitet, was bisherige Forschung widerspiegelt, da eine eher untergeordnete Rolle in der Behandlung aufgrund bisher fehlender Evidenzen für Effektivität und Verlässlichkeit postuliert wird (Bolten, 2013). Zur adäquaten Behandlung von FRS bestehen Leitlinien auf Grundlage von Expertenwissen, jedoch bislang ohne umfangreiche Evidenzstudien (z.B. AWMF, siehe Cierpka, 2014). Im Gegensatz zu allgemeinen Leitlinien bieten strukturierte Behandlungskonzepte oder -manuale eine konkretere Anleitung zur Behandlung. Jedoch ist anzumerken, dass auch hier eine schematische Evidenzprüfung mit direkt anwendbaren klinischen Studien von guter Qualität weitestgehend noch aussteht.

\section{Behandlung der FRS nach Konzepten oder Manualen}

Fast zwei Drittel der befragten Einrichtungen gaben an, nach einem Konzept oder Manual zu arbeiten. Am häufigsten wurde nach der IES / K-B / P nach Papoušek gearbeitet, welche an curriculare Fort- und Weiterbildungen geknüpft und evaluiert ist (Papoušek \& Wollwerth de Chuquisengo, 2006). So konnte beispielsweise in einer Studie mit 701 Familien bei rund $89 \%$ eine vollständige oder überwiegende Besserung der Symptomatik erreicht werden (Wollwerth de Chuquisengo \& Papoušek, 2004). Allerdings war diese Studie nicht als Therapieevaluation konzipiert, umfasste keine Kontrollgruppe sowie keine Randomisierung und ist somit nach den Empfehlungen der Agency for Healthcare Research and Quality (Manchikanti et al., 2014) als Evidenzklasse III einzuordnen. Die vorliegenden Ergebnisse ergaben die häufigste Anwendung dieses Konzepts in Beratungsstellen und Klini- 
ken. Das in dieser Studie zweithäufigste Beratungskonzept der EPB nach Ziegenhain, Fries et al. (2004) wird vor allem in Beratungsstellen angewandt. Im Gegensatz zu dem Konzept nach Papoušek ist dies eher ein niedrigschwelliger Beratungsansatz. Wirksamkeitsstudien beziehen sich bisher auf Risikogruppen hoch belasteter Mütter und zeigten einen kurzfristigen Effekt auf mütterliche Feinfühligkeit $(n=10$; Ziegenhain, Derksen \& Dreisörner, 2004). Jedoch konnten langfristige Effekte in einer Pilotstudie, die etwa der Evidenzklasse III entspricht, bisher nicht belegt werden (Pillhofer et al., 2015). Beide genannten Konzepte konzentrieren sich auf die Eltern-KindKommunikation und die Schaffung einer positiven Beziehung sowie auf die Förderung elterlicher Kompetenzen (Wollwerth de Chuquisengo \& Papoušek, 2004; Ziegenhain, Fries et al., 2004).

Als körperorientierte Konzepte wurden in der vorliegenden Studie vor allem die KKB nach Diederichs (2000) und die EEH nach Harms (2016b) genannt, welche beide hauptsächlich in Praxen angewandt wurden. Die Wirksamkeit dieser Konzepte wird zwar in ausgewählten Fallstudien beschrieben, jedoch stehen umfassende wissenschaftliche Wirksamkeitsstudien noch aus und sind daher als Evidenzklasse IV einzuordnen.

Insgesamt werden über 16 verschiedene Konzepte benannt, zusätzlich zu diversen eigenen Ausarbeitungen. Dieses Ergebnis steht im Einklang mit bisherigen Studien zur Versorgungslage (z.B. Cierpka, Stasch et al., 2007; FranzL, 2010). Viele dieser Behandlungskonzepte weisen gemeinsame Elemente und Überschneidungen auf, wie beispielsweise Psychoedukation oder Stärkung der elterlichen Kompetenzen. Es wird nur schwer ersichtlich, welche Teile davon wissenschaftlich evaluiert und an eine standardisierte, leitliniengerechte, curriculare Fort- und Weiterbildung gebunden sind (Ludwig-Körner, 2013).

Bestehende Wirksamkeitsstudien beleuchten vor allem die Eltern-Säugling/-Kleinkind Psychotherapie (s. z. B. Taubner et al., 2013; Windaus, 2012). Robert-Tissot et al. zeigten bereits 1996 anhand einer randomisiert kontrollierten Studie mit 75 Mutter-Kind Dyaden, dass verhaltenstherapeutische und psychoanalytische Ansätze frühkindliche dysfunktionale Beziehungsmuster im Kontext von FRS verbessern können. Allerdings stehen methodisch adäquate, klinische und longitudinale Wirksamkeitsstudien für die genannten Behandlungsansätze und -konzepte bezüglich der spezifischen Wirksamkeit bei FRS noch aus. Insgesamt zeigt sich hier nach wie vor ein deutlicher Forschungsbedarf, der sowohl national als auch international besteht (Gomez et al., 2004; Ludwig-Körner, 2018).

In Bezug auf die hohe Prävalenz der Schlafproblematik sowie deren Häufigkeit als Anmeldeanlass wäre eine Empfehlung, bereits bestehende hoch strukturierte, manualisierte und gut evaluierte Behandlungskonzepte für frühkindliche Schlafprobleme stärker in die Behandlung der FRS zu implementieren. Deren Wirksamkeit ist zudem international wie auch im deutschsprachigen Raum bereits wissenschaftlich bestätigt (z. B. Price et al., 2012; Schlarb \& Brandhorst, 2012; Schlarb et al., 2011).

\section{Limitationen}

Einige Einschränkungen sind bei vorliegender Studie zu berücksichtigen. So wurden die Daten ausschließlich anhand der Selbsteinschätzung des Fachpersonals in den Einrichtungen erhoben. Daher können professionell motivierte Antworttendenzen und verzerrte Angaben aufgrund sozialer Erwünschtheit sowie ungenaue Angaben aufgrund der persönlichen Schätzung nicht ausgeschlossen werden. Zudem ist die Rücklaufquote mit $35 \%$ sowie die finale Stichprobengröße mit 26\% der ursprünglichen Datenbank kritisch zu sehen. Bei Cierpka, Stasch und Kollegen (2007) war die Rücklaufquote mit $35 \% \mathrm{zu}-$ nächst vergleichbar, woraufhin eine telefonische Ruckrufaktion durchgeführt wurde, die die Rücklaufquote auf $50 \%$ ansteigen ließ. In der vorliegenden Studie erfolgte ebenfalls eine Erinnerung - jedoch via E-Mail. Zudem zeigen Daten aus der Online-Erhebung, dass 90 Teilnehmende die Umfrage zwar aufgerufen, jedoch unmittelbar abgebrochen haben. Zusammen mit der hohen Anzahl an unvollständigen Fragebögen deutet dies auf eine mangelnde Bereitschaft zur gewissenhaften Teilnahme hin. Eine mögliche Ursache hierfür könnte die Rekrutierung über das Internet sein. Diese machte persönlichen Kontakt unmöglich. Nach Jud et al. (2018) ist jedoch ein wiederholter persönlicher Kontakt für die Motivation zur Teilnahme äußerst förderlich, da diese vor allem durch die Arbeitsbelastung in den besagten Einrichtungen oftmals eingeschränkt ist.

Aufgrund der zum Teil offenen Antwortformate konnte zwar ein breites Spektrum an Angaben erfasst werden, jedoch erforderte die Auswertung, Antworten zusammenzufassen, was stellenweise mit einem Informationsverlust einhergehen könnte. Bisherige Forschung bestätigt, dass die Versorgungslage in Deutschland für FRS sehr breit gefächert ist (Cierpka, Stasch et al., 2007; FranzL, 2010; Ludwig-Körner, 2013; Taubner et al., 2013), sodass repräsentative, störungsspezifische, deutschlandweite Ergebnisse schwer abzubilden sind.

\section{Schlussfolgerung und Implikationen für die Praxis}

Kindlicher Schlaf ist ein Hauptanmeldegrund im Bereich der FRS. Es sollten über die Leitlinien hinaus konkrete 
Behandlungskonzepte und -manuale berücksichtigt werden, die in diesem Kontext gezielt das Schlafverhalten des Kindes andressieren. Weiterhin sollte sichergestellt werden, dass die Fachkräfte ausreichend in Bezug auf Wissen im Umgang mit Schlafproblematiken geschult sind. Aufgrund der Vielzahl an genannten Behandlungsansätzen sollte dringend weiter an einer wissenschaftlichen Überprüfbarkeit und leitliniengerechten Arbeitsweise sowie an einer Transparenz und einem Verständnis der Versorgungangebote gearbeitet werden.

\section{Literatur}

Arbeitsgemeinschaft der Wissenschaftlichen Medizinischen Fachgesellschaften (AWMF) (2015). Leitlinien zu (Nr. 028/041). Verfügbar unter: https://www.awmf.org/uploads(tx_szleitlinien/ 028-041__S2k_Psychische_Stoerungen_Saeuglinge_Kleinkind_ Vorschulalter_2017-10.pfd

Barr, R. G., Barr, M., Fujiwara, T., Conway, J., Catherine, N. \& Brant, R. (2009). Do educational materials change knowledge and behaviour about crying and shaken baby syndrome? A randomized controlled trial. Canadian Medical Association Journal, 180(7), $727-733$.

Bilgin, A. \& Wolke, D. (2017). Development of comorbid crying, sleeping, feeding problems across infancy: Neurodevelopmental vulnerability and parenting. Early Human Development, 109, 37 - 43. https://doi.org/10.1016/j.earlhumdev.2017.04.002

Bolten M. (2013). Infant psychiatric disorders. European Child \& Adolescent Psychiatry, 22 Suppl 1, S69-S74. https://doi.org/10. 1007/s00787-012-0364-8

Bolten, M. (2014). Regulationsstörungen im Kontext der ElternKind-Beziehung. Ars Medici-Pädiatrie, 1, 13-17.

Brisch, K. H. (2010). SAFE: Sichere Ausbildung für Eltern; sichere Bindung zwischen Eltern und Kind; für Schwangerschaft und erste Lebensjahre. Stuttgart: Klett-Cotta.

Bünder, P. \& Sirringhaus-Bünder, A. (2008). Elterliche Kompetenzen nachhaltig fördern mit Hilfe von Videoberatung: Die Arbeitsweise der Marte Meo-Methode. Praxis der Kinderpsychologie und Kinderpsychiatrie, 57(5), 330 - 345.

Cattarius, B. G. \& Schlarb, A. A. (2016) Gegenseitige Beeinflussung von Eltern und Babys in ihrem Schlafverhalten. Somnologie, 20, 189 - 198. https://doi.org/10.1007/s11818-016-0064-6

Cierpka, M., Hirschmüller, B., Israel, A., Jahn-Jokschies, G., von Kalckreuth, B., Knott, M., \& Windaus, E. (2007). Manual zur psychoanalytischen Behandlung von Regulationsstörungen, psychischen und psychosomatischen Störungen bei Säuglingen und Kleinkindern unter Verwendung des Fokuskonzeptes. In: M. Cierpka E. Windaus (Hrsg), Psychoanalytische SäuglingsKleinkind-Eltern-Psychotherapie. Konzepte-Leitlinien-Manual (S. 87 - 214). Frankfurt a. M.: Brandes \& Apsel.

Cierpka, M., Stasch, M. \& Groß, S. (2007). Expertise zum Stand der Prävention/Frühintervention in der frühen Kindheit in Deutschland. Köln: BzgA.

Cierpka M. (2014). Die unterschiedlichen Ansätze in Beratung und Therapie. In: M. Cierpka (Hrsg.), Frühe Kindheit 0-3 Jahre (S. 399 -414). Berlin: Springer.

Cohen, J. (1988). Statistical power analysis for the behavioral sciences (2nd ed.). Hillsdale, NJ: Erlbaum. http://doi.org/10. 4324/9780203771587
Cook, F., Mensah, F., Bayer, J. K. \& Hiscock, H. (2019). Prevalence, comorbidity and factors associated with sleeping, crying and feeding problems at 1 month of age: A community-based survey. Journal of Paediatrics and Child Health, 55(6), 644-651. https://doi.org/10.1111/jpc.14262

Covington, L. B., Armstrong, B. \& Black, M. M. (2018). Perceived toddler sleep problems, co-sleeping, and maternal sleep and mental health. Journal of Developmental and Behavioral Pediatrics, 39(3), $238-245$.

DeGangi, G. A., Breinbauer, C., Roosevelt, J. D., Porges, S. \& Greenspan, S. (2000). Prediction of childhood problems at three years in children experiencing disorders of regulation during infancy. Infant Mental Health Journal, 21(3), 156-175.

Diederichs, P. (2000). Der friedvolle Weg. Von der Anspannung in die Entspannung. Erfahrungen aus der Schreibaby-Ambulanz und ihre Bedeutung. In T. Harms (Hrsg.), Auf die Welt gekommen. Die neuen Baby-Therapien (S. $231-260)$. Berlin: Ulrich Leutner Verlag.

Diederichs, P. (2021). Methode und Anwendung der Ressourcenund Körperorientierte Krisenbegleitung. Verfügbar unter: https:// www.wikk.eu/index.php/methode-anwendung.

Dozier, M. \& Bernard, K. (2017). Attachment and biobehavioral catch-up: Addressing the needs of infants and toddlers exposed to inadequate or problematic caregiving. Current Opinion in Psychology, 15, 111-117.

Erickson, M. F. \& Egeland, B. (2004). Linking theory and research to practice: The Minnesota Longitudinal Study of Parents and Children and the STEEPTM program. Clinical Psychologist, 8(1), $5-9$.

FranzL - Fragen zur Lage (2010). Systemanalyse Interdisziplinäre Frühförderung in Bayern. Teil 1, Teil 2, Teil 3. Verfügbar unter: https://www.fruehfoerderung-bayern.de/fileadmin/files/PDFs/ FranzL_Resultate/Resultate_Teil_I.pdf; https://www.fruehfoer derung-bayern.de/fileadmin/files/PDFs/FranzL_Resultate/Re sultate_Teil_II.pdf; https://www.fruehfoerderung-bayern.de/fi leadmin/files/PDFs/FranzL_Resultate/Resultate_Teil_III.pdf

German Association of Infant Mental Health, GAIMH (2005). Präambel: Fort- und Weiterbildungsstandards für Begleitung, Beratung und Psychotherapie für Kinder von 0 bis 3 Jahren mit ihren Eltern und anderen Bezugspersonen. Verfügbar unter: https://www.gaimh.org/reader-veroeffentlichungen/positions papier.html

Gomez, C. R., Baird, S. \& Jung, L. A. (2004). Regulatory disorder identification, diagnosis, and intervention planning: Untapped resources for facilitating development. Infants \& Young Children, 17(4), $327-339$.

Hammerschmidt, U., \& Koch, J. (2018). Leitfaden Physiotherapie in der Pädiatrie. München: Elsevier.

Harms, T. (2016a). Körperpsychotherapie mit Säuglingen und Eltern. Grundlagen und Praxis. Gießen: Psychosozial-Verlag.

Harms, T. (2016b). Emotionelle Erste Hilfe. Grundlagen und Praxis bindungsbasierter Eltern-Säugling-Körperpsychotherapie. In T. Harms (Hrsg.), Körperpsychotherapie mit Säuglingen und Eltern (S. 143 -180). Gießen: Psychosozial-Verlag.

Hemmi, M. H., Wolke, D. \& Schneider, S. (2011). Associations between problems with crying, sleeping and/or feeding in infancy and long-term behavioural outcomes in childhood: A metaanalysis. Archives of Disease in Childhood, 96(7), 622-629. https://doi.org/10.1136/adc.2010.191312

IBM Corp. (2020). IBM SPSS Statistics for Windows, Version 27.0. Armonk, NY: IBM Corp.

Jud, A., Kosirnik, C., Mitrovic, T., Salah, H. B., Fux, E., Koehler, J. ... \& Knüsel, R. (2018). Mobilizing agencies for incidence surveys on child maltreatment: Successful participation in Switzerland and lessons learned. Child and Adolescent Psychiatry and Mental Health, 12(1), 3. https://doi.org/10.1186/s13034-017-0211-2 
Ludwig-Körner, C. (2013). Frühe Hilfen und Frühförderung: Eine Einführung aus psychoanalytischer Sicht. Stuttgart: Kohlhammer Verlag.

Ludwig-Körner, C., Kuchinke, L., Koch, G., Mattheß, J. \& Eckert, M. (2018). Eltern-Säugling-Kleinkind-Psychotherapie auf dem Prüfstand. Psychotherapeutenjournal, 17, 346-352.

Manchikanti, L., Falco, F. J., Benyamin, R. M., Kaye, A. D., Boswell, M. V., \& Hirsch, J. A. (2014). A modified approach to grading of evidence. Pain Physician, 17(3), E319-E325.

Nash, C., Morris, J. \& Goodman, B. (2008). A study describing mothers' opinions of the crying behaviour of infants under one year of age. Child Abuse Review: Journal of the British Association for the Study and Prevention of Child Abuse and Neglect, 17(3), 191 - 200. https://doi.org/10.1002/car.1017

Papoušek, M. \& de Chuquisengo, R. W. (2006). Integrative kommunikationszentrierte Eltern- Kleinkind-Psychotherapie bei frühkindlichen Regulationsstörungen. Praxis der Kinderpsychologie und Kinderpsychiatrie, 55(4), 235 - 254.

Pillhofer, M., Spangler, G., Bovenschen, I., Kuenster, A. K., Gabler, S., Fallon, B., Fegert, J. M. \& Ziegenhain, U. (2015). Pilot study of a program delivered within the regular service system in Germany: effect of a short-term attachment-based intervention on maternal sensitivity in mothers at risk for child abuse and neglect. Child Abuse \& Neglect, 42, 163 -173. https://doi.org/10. 1016/j.chiabu.2014.07.007

Price, A. M., Wake, M., Ukoumunne, O. C. \& Hiscock, H. (2012). Five-year follow-up of harms and benefits of behavioral infant sleep intervention: randomized trial. Pediatrics, 130(4), $643-$ 651. https://doi.org/10.1542/peds.2011-3467

Robert-Tissot, C., Cramer, B., Stern, D. N., Serpa, S. R., Bachmann, J. P., Palacio-Espasa, F. ... \& Mendiguren, G. (1996). Outcome evaluation in brief mother-infant psychotherapies: Report on 75 cases. Infant Mental Health Journal, 17(2), 97-114.

Sadeh, A., Juda-Hanael, M., Livne-Karp, E., Kahn, M., Tikotzky, L., Anders, T. F., Calkins, S. \& Sivan, Y. (2016). Low parental tolerance for infant crying: an underlying factor in infant sleep problems?. Journal of Sleep Research, 25(5), 501 - 507. https://doi. org/10.1111/jsr.12401

Sarimski, K. (2013). Soziale Risiken im frühen Kindesalter: Grundlagen und frühe Interventionen. Göttingen: Hogrefe Verlag.

Schlarb, A. A. \& Brandhorst, I. (2012). Mini-KiSS Online: an Internet-based intervention program for parents of young children with sleep problems - influence on parental behavior and children's sleep. Nature and Science of Sleep, 4, 41-52. https:// doi.org/10.2147/NSS.S28337

Schlarb, A. A., Brandhorst, I. \& Hautzinger, M. (2011). Mini-KiSS ein multimodales Gruppentherapieprogramm für Eltern von Kleinkindern mit Schlafstörungen. Zeitschrift für Kinder- und Jugendpsychiatrie und Psychotherapie, 39(3), 197-206. https:// doi.org/10.1024/1422-4917/a000106

Schlarb, A. A., \& Schneider, B. (2018). Schlaf im Säuglingsalter. Somnologie, 22(4), 273-284. https://doi.org/10.1007/s11818018-0186-0

Schmid, G., Schreier, A., Meyer, R. \& Wolke, D. (2010). A prospective study on the persistence of infant crying, sleeping and fee- ding problems and preschool behaviour. Acta Paediatrica, 99(2), 286-290. https://doi.org/10.1111/j.1651-2227.2009.01572.x

Schnatschmidt, M. \& Schlarb, A. (2018). Review: Schlafprobleme und psychische Störungen im Kindes- und Jugendalter. Zeitschrift für Kinder- und Jugendpsychiatrie und Psychotherapie, 46(5), 368 - 381. https://doi.org/10.1024/1422-4917/a000605

Spranz-Fogasy, T. (2010). Verstehensdokumentation in der medizinischen kommunikation: Fragen und Antworten im Arzt-Patient-Gespräch.In A. Deppermann, U. Reitemeier, R. Schmitt, T. Spranz-Fogasy (Hrsg.), Verstehen in professionellen Handlungsfeldern (S. 27 -116). Tübingen: Narr.

Taubner, S., Munder, T., Unger, A. \& Wolter, S. (2013). Zur Wirksamkeit präventiver Früher Hilfen in Deutschland-ein systematisches Review und eine Metaanalyse. Praxis der Kinderpsychologie und Kinderpsychiatrie, 62(8), 598-619.

Weiß, H. \& Sann, A. (2013). Interdisziplinäre Frühförderung und Frühe Hilfen - Wege zu einer intensiveren Kooperation und Vernetzung. Köln: NZFH.

Windaus, E. (2012). Die Ergebnisforschung zur Säuglings-/Kleinkind-Eltern-Psychotherapie. In M. Cierpka (Hrsg.), Frühe Kindheit $0-3$ (S. 503-512). Berlin: Springer.

Winsper, C. \& Wolke, D. (2014). Infant and toddler crying, sleeping and feeding problems and trajectories of dysregulated behavior across childhood. Journal of Abnormal Child Psychology, 42(5), 831 - 843. https://doi.org/10.1007/s10802-013-9813-1

Wollwerth de Chuquisengo, R. \& Papoušek, M. (2004). Das Münchner Konzept einer kommunikationszentrierten ElternSäuglings-/Kleinkind-Beratung und-Psychotherapie. In M. Papoušek, M. Schieche \& H. Wurmser (Hrsg.), Regulationsstörungen der frühen Kindheit (S. 281 -309). Bern: Huber

Zero to Three (2016). DC: 0-5: Diagnostic classification of mental health and developmental disorders of infancy and early childhood. Washington, DC: Zero to Three.

Ziegenhain, U., Derksen, B. \& Dreisörner, R. (2004). Frühe Förderung von Resilienz bei jungen Müttern und ihren Säuglingen. Kindheit und Entwicklung, 13(4), 226 -234. https://doi.org/10. 1026/0942-5403.13.4.226

Ziegenhain U., Fries M., Bütow, B. \& Derksen, B. (2004) Entwicklungspsychologische Beratung für junge Eltern. Kindheit und Entwicklung, 13, 226 - 234. https://doi.org/10.1026/0942-5403. 13.4.226.

\section{Förderung}

Open Access-Veröffentlichung ermöglicht durch die Universität Bielefeld.

\section{M.Sc. Marisa Schnatschmidt}

Fakultät für Psychologie und Sportwissenschaft

Abteilung für Psychologie

Universität Bielefeld

Postfach 100131

33501 Bielefeld

Deutschland

marisa.schnatschmidt@uni-bielefeld.de 